\title{
Educación física en el proceso de modernización
}

\section{Prácticas e ideales ${ }^{1}$}

\section{Victor Jairo Chinchilla Gutierrez}

En este documento se presentan algunos resultados de la investigación histórica realizada durante los años 1999 y 2001 con el objetivo de establecer la manera como se configuró la educación tísica durante el proceso de modernización, en la primera mitad del siglo XX en Colombia. Se buscó dilucidar los significados sociales y políticos de su práctica, expresados en ideales relacionados con e] proyecto político de la modernización. De esta manera se muestra cómo la educación física cumple un papel político de formación y difusión de estos ideales en la institución escolar y otros ámbitos sociales del país.

Palabras clave: Educación física, modernización, cultura, cultura política, prácticas, discursos, sociedad, orden, poder, identidad, raza, cuerpo, salud, higiene.

El análisis se hace desde la cultura política para poner en evidencia que el proyecto de control y educación del cuerpo se relaciona con las modificaciones en la organización social y las costumbres, en el marco de condiciones políticas y sociales que permitieron una pedagogía que centra su interés en ejercicios y prácticas corporales de las cuales se esperan resultados en términos de adhesión al provecto político dominante.

En este orden de ideas, es valido preguntarse por el cómo y con qué significado se apropiaron prácticas como la gimnasia, los deportes, danzas y juegos; qué sentido se asignaba a las ceremonias, desfiles y competiciones masivas; de qué manera los discursos y prácticas específicas que tienen lugar en el campo de la educación física, dejan entrever cierto tipo de concepciones sobre la sociedad, el orden establecido y el lugar que los individuos y los distintos grupos sociales ocupan dentro de él.

La investigación se realizó desde un enfoque histórico, cultural y social, y se utilizaron como fuentes: legislación, documentos políticos de los gobiernos, informes de ministros de Educación, documentos y tesis académicas, textos guía, programas y manuales escolares, informes de instituciones y relatos de vida de personas de la época. El análisis de los documentos permitió ubicar aquellos descriptores que de manera explicita se relacionan con los referentes de la cultura política como son los conceptos orden, poder, identidad, raza, formación social, disciplina, moral, salud e higiene pública, aptitud, educación. En una fase posterior se procedió a establecer relaciones, destacando convergencias e identidades que pusieron en evidencia los ideales de la educación física en el proceso de modernización. Estos ideales, son el tema del presente artículo.

\section{Educación física, prácticas corporales V sociedad}

La educación física se basa en una intervención educativa sobre el cuerpo y desde el cuerpo mediada por actividades físicas, lúdicas y deportivas, portadoras de valores y

\footnotetext{
${ }^{1}$ El artículo reúne parte del informe de investigación realizada en el grupo de investigación, educación y cultura política, adscrito a la maestría de historia de la educación y la pedagogía

* Profesor de la facultad de Educación Física. Universidad Pedagógica nacional
} 
significados propios de cada tipo de sociedad y momento histórico. Integra diferentes prácticas e ideas sociales que tienen su realización en la corporalidad del hombre. Cambia de acuerdo con la dinámica de la sociedad y da respuesta a transformaciones de la cultura, que destaca las prácticas como actividades, medios o fines.

La manera como se establecen las relaciones entre educación física y sociedad puede ser abordada desde la forma como las prácticas corporales o motrices se constituyen en cultura y por lo tanto, además de ser expresión de ella, intervienen en su consolidación y transformación. Diferentes autores, en distintas áreas del conocimiento, proporcionan valiosos aportes para orientar la búsqueda apropiada a lo largo del proceso de investigación. De ellos se presenta una breve síntesis, que justifica y fundamenta la construcción del concepto de cultura política y su relación con la educación física.

Marcel Mauss aporta el concepto de técnicas del cuerpo ${ }^{2}$. Esas técnicas se entienden como "todo acto tradicional eficaz", de carácter práctico y construido, aceptado y legitimado por su eficacia social. Por ejemplo, las técnicas de nacimiento, de comida, de saludo, de relación sexual, de uso del tiempo y del espacio, y de formas de interacción social . Las técnicas del cuerpo son específicas de cada sociedad, producto de una labor de la razón práctica colectiva que conforman hábitos que varían con los individuos y sus limitaciones, con las sociedades, las reglas de urbanidad, la educación o la moda.

Desde otra perspectiva sociológica, Luc Boltansky desarrolla el concepto de usos sociales del cuerpo $^{3}$, para explicar los valores y prácticas mediante los cuales la sociedad utiliza el cuerpo, para sus intereses políticos, ideológicos, comerciales o sexuales. Según Boltansky; cada sociedad construye un determinado uso del cuerpo definido como el conjunto de relaciones que los individuos mantienen con el suyo y que expresan las características propias de los grupos sociales en un momento histórico determinado. Los usos sociales del cuerpo incluyen imágenes, ideologías, políticas, aspectos estéticos, sexuales, de salud y de cuidado que se expresan en la convivencia social y generan una amplia producción de prácticas, actitudes y reglas de conducta que difieren entre las clases sociales.

En la perspectiva histórico-social del desarrollo científico de la educación física, el filósofo alemán Kurt Meinel expone el papel del movimiento y del lenguaje en la estructuración del pensamiento, y el desarrollo de las fuerzas del trabajo en los procesos de socialización. "El proceso de invención, elaboración y constante mejoramiento de los instrumentos de trabajo es inconcebible fuera del contexto de una valoración y aplicación continua de nuevas prácticas de movimiento, resultantes de una labor colectiva"4. El desarrollo de las fuerzas productivas, desde la herramienta y la máquina hasta la alta tecnología, se fundamenta en la búsqueda de perfeccionamiento de la acción humana, de tal manera que en su evolución histórica los diferentes movimientos se corresponden con el desarrollo de las fuerzas productivas y la solución de las necesidades del hombre para su desenvolvimiento social.

Norbert Elías explica el fenómeno del deporte y la actividad física, correspondiente a la naturaleza dinámica y relacional de los seres humanos y de sus sociedades, que no admiten separación entre la naturaleza y la cultura, cuya complejidad debe ser entendida

\footnotetext{
${ }^{2}$ Marcel Mauss. Las Técnicas de! cuerpo. Citado por: Barreau y M orne (1991). Epistemología e antropología del deporte. Madrid, Alianza Editorial, pp. 213-227.

${ }^{3}$ Lun Boltanskv: Les usages sociaux du corps. En: Epistemología y antropología del deporte, Jean Jacques Barreau, Jean Jaques Morne. P 217-218

${ }^{4}$ Kurt Meinel. Didáctica del movimiento, p. 17. 
en una totalidad que no acepta dualismos ${ }^{5}$. Para Elías, es importante preguntarse qué clase de sociedad es ésta en la que cada vez más gente utiliza parte de su tiempo libre en practicar y observar como espectadores estas competiciones no violentas de habilidad y fuerza corporal que llamamos "deporte".

Pierre Parlebas, investigador francés, introduce en el campo de la educación física los conceptos de sociomotricidad y etnomotricidad, a partir de los cuales caracteriza la significación de las prácticas motrices y su uso particular por los grupos sociales ${ }^{6}$. La sociomotricidad explica las diferentes normas, roles, prescripciones, que producen las acciones motrices de los grupos sociales; y la etnomotricidad expresa las características de las prácticas corporales de cada época, de las normas y los valores relacionadas con el cuerpo en distintas culturas. En los dos casos las acciones motrices adquieren significación social y cultural, determinadas por las condiciones y características del medio en donde se realicen.

\section{Cultura política y educación física}

Con apoyo en Clifford Geertz, quien entiende la cultura como un entramado de significados que otorgan sentido a una acción humana, y que "el análisis de la cultura ha de ser por lo tanto, no una ciencia experimental en busca de leyes, sino una ciencia interpretativa en busca de significaciones... de expresiones sociales que son enigmáticas en su superficie" ${ }^{\prime \prime}$, se puede afirmar que la cultura política se conforma a partir de una red de significados generados en prácticas y saberes sociales que expresan las ideas, sentimientos, valores de individuos y grupos sociales, respecto a su pertenencia e identidad con una comunidad y las acciones que de ello se derivan. En esta red de relaciones, el cuerpo, el juego, el deporte y el conjunto de producciones culturales de la actividad física, expresan en su lenguaje, las significaciones de cultura política de la sociedad en un periodo histórico determinado.

En la acción corporal del ser humano no se produce separación entre la dimensión biológica y psicológica o individual y social. El hombre actúa como unidad, en un contexto donde las prácticas y los gestos se realizan de acuerdo con un conjunto de significaciones que le otorgan sentido a la acción. "Cuando el hombre actúa lo hace íntegramente sin

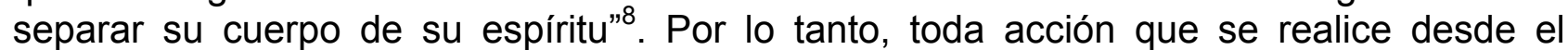
cuerpo o hacia él, tiene sentido y significado, y manifiesta ideas, valores o actitudes sobre el hombre y la sociedad.

Desde estos aportes teóricos se identificaron los tres grandes ideales de la educación física en el proceso de modernización: el rnens sana in corpore sano responde a la manera como se conjugaban la salud, la moral, la raza y la higiene; el de orden; disciplina se relaciona con la acción de la educación física en la identidad nacional, el orden social y la formación cívica; e! práctico, referido a la formación de habilidades y destrezas correspondientes con las transformaciones en el trabajo, el uso del espacio, el tiempo y la distribución de la actividad social.

La explicación de dichos ideales se realiza a partir de la contextualización en la modernización y la escolarización de la educación física, además establecen los componentes conceptuales, las prácticas y sus significados. En el manejo temporal, se

\footnotetext{
${ }^{5}$ Norbert Elías Y Eric Dunning, Deporte y ocio en el proceso de la civilización. P.20

${ }^{6}$ Pierre Parlebas, Jeux sports et siciétés. Lexique de praxéologie motrice, pp.341,343

${ }^{7}$ Clifford Geertz La interpretación de lasculturas. P.20

${ }^{8}$ Ommo Gruppe Teoría, pedagogía y antropología de la Educación Física 
busca identificar el momento del surgimiento It evolución en las tres primeras décadas y su desarrollo en la cuarta y quinta décadas del siglo $\mathrm{XX}$.

\section{Modernización y educación física}

El proceso de modernización en Colombia puede interpretarse desde tres grandes dimensiones propuestas por Jorge Orlando $\mathrm{Melo}^{9}$, en las cuales se gesta la educación física. Para Melo, las rápidas transformaciones del país se producen, en primer lugar, en el terreno político con la movilización social, en particular de las décadas de los años 30 y 40, que junto con la aparición de los medios de comunicación de masas y la expansión del sistema educativo masivo, hacen imposible un regreso del autoritarismo de orden tradicional. En segundo lugar, en el campo de la cultura, manifestado por el desarrollo de un sistema escolar masivo, la aparición del mercado cultural nacional, la creación de una práctica científica continua y la divulgación del conocimiento científico; y en tercer lugar, en el terreno económico, con la evidente consolidación del capitalismo y la eliminación acelerada de las formas de producción precapitalista.

Las migraciones y el acomodamiento en los espacios urbanos producen problemas relacionados con la higiene y salud pública ${ }^{10}$, el uso del tiempo'11, las relaciones cotidianas $^{12}$, la organización del transporte, el acceso a la cultura y la educación, las actividades de ocio, el uso de espacios y la regulación de comportamientos conforme a nuevas normas de convivencia.

En este contexto se genera un discurso pedagógico y político sobre la educación del individuo y la formación de la nación, del que forma parte un conjunto de saberes y prácticas sobre la corporalidad. Al respecto, la antropóloga Zandra Pedraza ${ }^{13}$ expone que, mientras la sociedad señorial se basaba en el discurso de la urbanidad y de la moral, el proceso de modernización, y dentro de él, el desarrollo del capitalismo, genera el discurso de la salud y el de la educación física, asignando a éste último el papel activo de intervenir el cuerpo como medio de formación de finalidades y propósitos de organización de la vida social. Pero, si bien la técnica y la organización de estas prácticas correspondían a un discurso moderno, muchas de las ideas que las sustentaron se fundamentan en valores e imaginarios anteriores, de carácter tradicional. Se inicia así la tarea de la educación física al interior de la escuela, que se entiende como la institución clave en el proceso modernizador.

La organización de la educación física en la escuela comprende un largo proceso que se inicia con las recomendaciones generales y prácticas espontáneas o dirigidas para realizar en determinados momentos de la jornada, hasta el establecimiento de horarios, programas, actividades, formas de organización y construcción de escenarios que la consolidaron como asignatura escolar ${ }^{14}$.

\footnotetext{
${ }^{9}$ Jorge Orlándomelo. Algunas consideraciones globales sobre "modernidad” y "modernización” . en: Colombia: El despertar de la modernidad, Ediciones Foro Nacional por Colombia, 5. Edición., 1998

${ }^{10}$ Noguera Carlos Ernesto, (1998)." La higiene como política". En: Anuario colombiano de historia social y de la cultura, No. 25 Universidad nacional, Facultad de Ciencias Humanas, Departamento de Historia, Bogotá. P. 188-215

${ }^{11}$ Archilla Mauricio, (1991). El uso del tiempo libre de los obreros. En: Anuario colombiano de historia social y de la cultura. U.N. No. 18 y 19, 1990-1991, Pp. 145,184

${ }^{12}$ ALVAREZ, Alejandro, NOGUERA, Carlos Ernesto. Op. Cit.

${ }^{13}$ PEDRAZA, Sandra. (1999). En cuerpo y alma. Visiones del progreso y de la felicidad. Universidad de los Andes. Bogotá

${ }^{14}$ URIBE, Antonio José. Op.cit. P. 76-77 
La inclusión de la educación física en la escuela es considerada como uno de los logros de la pedagogía activa ${ }^{15}$ por sus características prácticas que posibilitaron la apertura de nuevos espacios de actividad y lúdica para la formación del niño.

En tanto lo físico, lo moral y lo intelectual fueron concebidos como una unidad orgánica indivisible y de influencias recíprocas, la educación física comenzó a ser pensada como la base de la salud intelectual y moral. Al régimen claustral de privación de aire y de luz, además de la exigencia de quietud de la escuela tradicional, se le contrapone una institución centrada en el movimiento del alumno. El desarrollo físico natural Y equilibrado, meta de la nueva educación física, se convierte en el fundamento del desarrollo normal de las actividades de orden superior ${ }^{16}$.

Pese a los avances en la educación física, no se superó la diferenciación entre el campo y la ciudad.

\begin{tabular}{|l|l|}
\hline MODERNIZACIÓN & EDUCACIÓN FÍSICA \\
\hline Transformaciones en el modo de vida & Mens sana in corpore sano \\
Secularización: de la moral cristiana a la & \\
moral biológica & \\
Problemas de salud e higiene & \\
Fortalecimiento y vigor de la raza & \\
Cuerpo y moral social & \\
\hline $\begin{array}{l}\text { Orden social. Disciplina colectiva } \\
\text { Identidad nacional. Símbolos y espectáculos }\end{array}$ & Orden, identidad y disciplina \\
Raza & \\
\hline Transformaciones en el ámbito económico & Nuevas prácticas y técnicas del cuerpo \\
Habilidades y destrezas & \\
Productividad. Técnicas de trabajo & \\
Cambios de comportamiento social & \\
\hline
\end{tabular}

Mientras a los niños del campo se asignaban actividades relacionadas con la higiene, la disciplina y el orden, a los alumnos de las escuelas urbanas se interesaban por prácticas activas como el deporte ${ }^{17}$. En la práctica también se dio una división por razones económicas y de grupos sociales, que se expresó en mayores posibilidades de los alumnos de colegios de mejores recursos económicos, mientras que los niños más pobres no tuvieron el acceso a la práctica de la asignatura, o a determinados tipos de prácticas relacionadas con algunos deportes.

En la mayoría de las regiones del país, la escuela era una casa poco adecuada para las labores de la enseñanza, en donde los niños se acomodaban en bancos o troncos, con la pizarra y el gis. La actividad física se realizaba como parte de la vida cotidiana, pues en la mayoría de los casos los niños realizaban un extenso recorrido entre la escuela y su casa, por caminos en los que se jugaba, se trepaba a los árboles y se llevaban los mandados o encargos que se solicitaban en sus hogares ${ }^{18}$.

Aún así, en el contexto general de la política educativa, la educación física se va consolidando como asignatura de formación y como principio general de formación, con un papel muy preciso en los cambios exigidos por las nuevas circunstancias políticas y sociales.

\footnotetext{
${ }^{15}$ SAENZ, Javier, SALDARRIAGA, Oscar y OSPINA, Armando, Mirar la infancia: Pedagogía, moral y modernidad en Colombia, 1903-1946, Vol. 2, Colciencias, Bogotá, 1997, p. 428

16 Ibid, p.57

${ }^{17}$ En el decreto 491 de 1903 sobre plan de estudios se puede observar esta diferenciación

${ }^{18}$ Entrevista a Bernardo Chinchilla Chivatá, escolar de la época, Zipaquirá, diciembre de 1999 
La relación entre los cambios generados en el proceso de modernización y la educación física, se resume en el cuadro anterior. En la primera columna, se puede apreciar el eje de las grandes transformaciones, y en la segunda, la manera como éstas transformaciones se integran en los ideales de la educación física.

\section{Los ideales de la educación física}

El informe presentado por el Instituto San Juan de Córdoba a la Comisión Nacional de educación física, es un buen ejemplo de la manera como se justificaba y asignaba la finalidad que debía cumplir la educación física en el marco de los procesos de modernización $^{19}$

Se mencionan en el citado informe, los tipos de ejercicios y propósitos del programa de educación física en las instituciones educativas. Este comprendía los siguientes aspectos:

1. Ejercicios destinados a producir el efecto higiénico, con los cuales se obtiene una actividad mayor de las grandes funciones orgánicas, en particular la circulación y la respiración.

2. Ejercicios destinados a producir el efecto correctivo, mediante movimientos bien determinados que localicen su acción sobre los músculos débiles del dorso, del abdomen, de las piernas y de la cabeza.

3. Ejercicios destinados a producir un resultado económico, es decir, a que el alumno aprenda a medir y conocer sus fuerzas para que no las malgaste, perfeccione su marcha normal y produzca el máximo trabajo con el mínimo de fatiga; y

4. Ejercicios destinados a producir una influencia moral y psíquica. Estos no son diferentes a los enunciados, sino que mediante la modulación y asociación de los mismos se producen efectos psicológicos como el placer, la animación, y las cualidades morales elevadas, indispensables para la educación integral de los educando.

El programa reúne los fundamentos teóricos y pedagógicos de orden biológico, social, técnico y moral que manifiestan los ideales de la educación física colombiana en la primera mitad el siglo $\mathrm{XX}$, orientados al fortalecimiento físico para tener un cuerpo sano en una mente sana, al orden y la disciplina y al desarrollo de habilidades y destrezas de carácter técnico.

Los ideales se expresan a través de prácticas y discursos, organizados de tal forma que a la vez que son parte de procesos culturales y sociales, buscan unos determinados efectos formativos sobre la sociedad, en donde el cuerpo se toma como fin, medio u objeto. Cada ideario conforma un campo en el cual confluyen diferentes componentes que corresponden a prácticas y discursos que han sustentado la educación física a través de su evolución histórica.

\section{Fortalecimiento físico: mens sana in corpore sano}

El ideal del fortalecimiento físico se universa-liza en el precepto mens sana incorpore sano, pese a los desplazamientos producidos por el proceso de modernización, se mantiene justificado en argumentos tanto religiosos y morales, como de carácter

\footnotetext{
${ }^{19}$ Instituto San Juan de Córdoba (1934) Informe de La Comisión Nacional de Educación Física, san Juan de Córdoba 
biológico, psicológico y pedagógico. Sus discursos, prácticas y métodos se orientan hacia procesos de formación integral.

En la apropiación de este precepto $^{20}$, compartido por diferentes corrientes pedagógicas, tanto de la pedagogía católica como de la activa, se expresan ideas sobre la salud del cuerpo, y sus relaciones con la salud del alma y la del cuerpo social, a través de la integración entre la formación corporal y la formación moral, dirigido a lograr un comportamiento social adecuado en aptitudes y en hábitos. En la conformación de este ideal social se interrelacionan discursos de carácter fisiológico y médico con discursos morales de virtud y carácter, orientados a satisfacer necesidades de salud pública, de higiene, de fortalecimiento de la raza y armonía social, desarrollados en la práctica a través de estrategias de higiene, fortalecimiento de las ciencia médicas y biológicas, divulgación de la eugenesia, empleo de técnicas biotipológicas que toman como escenario de promoción la educación pública, y confluyen en la educación física.

El precepto mens sana in corpore sano se basa en la idea de que la educación física, o educación del cuerpo, tiene por objeto conservar la salud, acrecentar la fuerza y la agilidad de los miembros, contribuyendo a la educación moral. Su necesidad resulta de la unión íntima del alma con el cuerpo. Los órganos corporales son los instrumentos de las acciones exteriores; de aquí la necesidad de que sean perfeccionados para atender lo mejor posible a su destino. Por otra parte, el alma tiene necesidad de órganos corporales para adquirir capacidades intelectuales, pues si el organismo funciona mal, la vida intelectual se encuentra ordinariamente decaída ${ }^{21}$.

La educación física, basada en el canon de rnens sana in corpore sano, se convierte en una estrategia para afrontar el problema de la salud que exige atención prioritaria, tanto a nivel social como al interior de la organización de la escuela. Al comenzar el siglo y como consecuencia de la miseria producida por la guerra de los Mil Días, que produjo enfermedades e intensificó victos como el alcoholismo; en la constante amenaza de las enfermedades tropicales como parasitismo, anemia y paludismo; en el sedentarismo de sectores de clase medias y altas; en la mala alimentación y la desnutrición; en la falta de higiene; en el crecimiento de la urbanización, fue necesario impulsar programas y campañas de salud que tenían su origen en la escuela como institución pública que facilitaba llegar a toda la población.

A la educación física se le asigna el objetivo de prevenir gran número de enfermedades, lo que la convierte en un método de educación higiénica cuyo fin es educar en la nutrición y el cuidado del cuerpo para el aumento de la fuerza y la resistencia del individuo. Se reconoce que el ejercicio muscular conserva la fuerza de los provistos y proporciona energía vital a los débiles.

También corresponde a la educación física la formación de hábitos de orden moral para alejar al alumno del camino de la corrupción, pues el ejercicio despierta una constante preocupación por sí mismo y por evitar todo lo que pueda ser pernicioso al buen estado y conservación del cuerpo. Como agente de orden moral, tiene como condición el buen funcionamiento del organismo.

\footnotetext{
${ }^{20}$ El origen del precepto mens sana in corpore sano y su apropiación en Colombia ha sido objeto de análisis por Hernán Felipe Prieto, quien clarifica su origen latino y no griego. Sobre el sentido dado por Juvenal en su sátira $X$, y la posterior propagación dentro del dualismo cristiano. Ver: "Mente sana en cuerpo sano ', en Documentos centrales de la Conferencia Latinoamericana de la Educación física - y Sociedad. Bogotá. ACPEE, 1992. pp. 51-78

${ }^{21}$ Hermana Antonia Isabel. (1933). "Necesidad de la Educación Física". En: Revista La Escuela Primaria. Imprenta del Departamento, Bucaramanga. p. 278-279.
} 
La educación física debe andar paralelamente con la educación moral, de modo que se empleen tanto en el alma como en el cuerpo todos los cuidados que sus necesidades reclaman. Sin esta armonía de las dos educaciones, la obra sería incompleta, frustrada, y tanto la joven como el niño, volverían a la sociedad y al seno de las familias, incapaces de realizar y llenar el objeto para el cual Dios los ha puesto en este mundo. Además es importante la educación física, pues si el vigor físico no produce el intelectual y moral, por lo menos lo conserva y acrecienta. No se puede negar que lo moral influye sobre lo físico y viceversa. La experiencia prueba la veracidad del aforismo: "alma sana en cuerpo sano"22.

El propósito de formación moral buscaba actuar sobre las cualidades de la personalidad de la raza, que combina la vivacidad, sensibilidad, espíritu fogoso y enamorado, con cierta incapacidad para el ejercicio sostenido y metódico; carencia innegable de aptitudes para el orden práctico y una incompleta organización de la voluntad que se manifiesta en incapacidad para la acción ${ }^{23}$.

La polémica sobre la raza es el centro del debate sobre la población y el orden social, que se desarrolla durante la primera parte del siglo XX. En los primeros años del siglo, se plantea la tesis de la degeneración de la raza, y se ve en la educación física el remedio que impida, y aún, que compruebe la falsedad que hay en esa afirmación.

La acción de la educación física en el fortalecimiento de la raza va integrada con la atención a la moral y la higiene para formar actitudes en el trabajo y la vida social. Para ello se ve al deporte como el modelo ideal de práctica, en el cual se pueden ver la fuerza, la entereza y la voluntad de una raza triunfadora ${ }^{24}$.

La atención al fortalecimiento racial produjo una serie de métodos e instrumentos de medición para establecer los parámetros de normalidad-anormalidad, que se constituyó en una de las principales actividades de los médicos escolares y posteriormente de los educadores. Se desarrolla la biotipología, como disciplina que estudia los tipos humanos. Sus conceptos y técnicas se divulgaron por diferentes medios e hicieron parte de los programas de capacitación y formación de profesores de educación física y de los programas de higiene.

Estos conceptos fueron apropiados por los colegios, en donde se consideraba a la educación física como un principio y base fundamental del desarrollo del individuo en todas sus actividades; como la fuente del progreso de los pueblos y de la fortaleza y mejoramiento de la raza ${ }^{25}$

Una de las estrategias para el fortalecimiento racial fue la creación de las Colonias Escolares para restablecer el equilibrio fisiológico de los niños, perturbado por deficientes condiciones de vida y enfermedades tropicales mal tratadas. Desarrollan una verdadera defensa de la raza que se complementa con la labor propiamente educativa $^{26}$. Los muchachos se sometían a un régimen de vida sana y amable que influye en poco tiempo en el mejoramiento de la curva de peso y en la conducta. En la colonia se continúa con la labor de enseñanza y se educa en hábitos de vida saludable y constructiva y en oficios acomodados a sus capacidades físicas para hacerlos aptos

\footnotetext{
${ }^{22}$ Colegio Nuestra señora de la consolación. (1934). Informe a la comisión nacional de educación física, toro, valle

23 Ibid.

${ }^{24}$ LOPEZ, Miguel Jiménez "La educación moral”, En: La escuela y la vida, op. Cit p..214

${ }^{25}$ Colegio Ricaurte (1934) Informe a la Dirección de educación física, Bogotá, julio

${ }^{26}$ Ministerio de Educación Nacional. Informe del Ministerio. Bogotá, 194, p.69-83
} 
para la vida de la comunidad. Su ubicación se hizo teniendo en cuenta los factores de clima, la cercanía a los poblados que permitiera el aprovisionamiento, así como la atención médica y dental. Estaban a cargo de personas de reconocida competencia y de maestros pagados por los departamentos. Allí el alumno podía permanecer durante una temporada e incluso prolongar su estadía de acuerdo con la prescripción médica.

\title{
Orden social, identidad y disciplina
}

El segundo ideal de la educación física se orienta al orden social y la disciplina. Se trataba de formar hombres aptos para la lucha por la vida, ciudadanos vigorosos que trabajaran por el progreso y el bienestar del pueblo, hombres que guardaban en sí las características de trabajador y de guerrero. Las naciones derivan un singular provecho de la educación física en sus ciudadanos. La moralidad y la virtud de ellos es el reflejo de una buena educación, a la par que motivo de un buen nombre. La buena salud es fuente de alegría para el hogar y un excitante al trabajo y a la actividad. Se promovió la idea de que la educación física es la base para formar las cualidades del buen ciudadano.

\begin{abstract}
Ciudadanos vigorosos son causa del progreso y bienestar de un pueblo; con ellos habrá brazos fuertes y robustos, ya para manejar el azadón, ya para llevar el arma. Nada hay que contribuya más a hacer una nación fuerte en la guerra y en la paz, como la salud $y$ virtudes de sus hombres. Sin proclamar las excelencias de la guerra, sí diremos que es bueno estar listos para ella, y que el mejor apercibimiento es ir preparando soldados fuertes y vigorosos que puedan resistir la inclemencia de los climas o la rudeza de las marchas ${ }^{27}$.

Uno de los componentes del ideal de orden son los actos deportivos masivos, como la realización de revistas gimnásticas y de torneos deportivos, juegos y encuentros. Eran antecedidos por rituales patrios, discursos en los que se relacionaba la actividad con la grandeza de la patria, de la raza o de la nación. La población contemplaba absorta y respetuosa estas celebraciones. Con ello se fueron afirmando en la conciencia social elementos de identidad y pertenencia simbólica a una patria a través de comportamientos sociales, actitudes y patrones culturales que reforzaban la autoridad compartida del poder civil, eclesiástico y militar
\end{abstract}

En los años treinta las grandes demostraciones masivas toman fuerza en el mundo entero, pero especialmente en la Alemania regida por el nacional socialismo y fortalecida con la celebración de los Juegos Olímpicos de Berlín en 1936. Se fortalecen en Colombia las actividades masivas a través de la ley de fiestas cívicas como el Día de la raza, la Fiesta de la Juventud, el Día Olímpico, la Semana Bolivariana, que se agregaron a las festividades patrias y religiosas tradicionales. Se establece además, la "institución a la bandera" como un acto de cultura cívica y recompensa de los méritos intelectuales y morales de la juventud, así como el culto a los símbolos de la nacionalidad y formación de auténticos patriotas ${ }^{28}$.

Los ejercicios de fuerza se consideraban sinónimos de ciencia y salud, de buena educación, de patriotismo, de virtudes cívicas, de "no querer para otro lo que no queramos para nosotros", de caridad; que "será la victoria decisiva de las grandes naciones", de las naciones mejor educadas en la ciencia, en el arte y en la higiene, en su lucha permanente por la paz universal y el triunfo definitivo del hombre fiel y veraz ${ }^{29}$.

\footnotetext{
${ }^{27}$ BEJARANO, Jorge. La educación Física, pp. 43-44

${ }^{28}$ Decretos 2216 de 1983; 275 de 1939; 1568 de 1940; 784 de 1941; 1165 de 1941; 738 de 1942; 449 de 1945; 2229 de 1947; 1140 de $1947 ; 31$ de 1948

${ }^{29}$ Ibid. P. 7.
} 
Se promovió la idea de llevar a la juventud al estadio, al aire libre y al sol, para avasallar los instintos primarios en el ejercicio conciente de la fuerza, cimentar el carácter, la voluntad, la lealtad y el juego limpio en las competencias deportivas, combatir la degeneración y el alcoholismo, prolongar esta acción hasta las zonas pobres de la población para difundir la salud, que como se ha dicho, es el único capital de los trabajadores. Este es el vasto empeño del Gobierno, que aspira a formar una conciencia nacional y una organización de la cultura física capaces de tutelar e incorporar a la raza ${ }^{30}$

A veces se combinaban los desfiles con demostraciones gimnásticas y presentaciones folclóricas. Con ello se pasaba un día cívico y se recordaba la pertenencia a la patria mientras se regresaba a la vida cotidiana. Se buscaba el contacto físico en el espacio público, en donde se moldean los cuerpos en emociones comunes que refuerzan la adhesión al Estado-nación.

Los esfuerzos de formación pedagógica no sustituyeron la influencia militar que se mantuvo de manera directa con militares docentes, pero principalmente a través de una mentalidad autoritaria y directiva aplicada desde las tendencias pedagógicas de la educación física. Este es un tema que no se puede calificar de manera unilateral, pues dentro de algunos puntos de vista sobre las prácticas de formación militar se concibe al oficial como un pedagogo preocupado por la educación física integral. En el año de 1941, se decreta la instrucción militar en los colegios de segunda enseñanza, escuelas normales e industriales, con una intensidad de cuatro horas semanales, que sustituyen la clase de educación física y se realizan por instructores militares. ${ }^{31}$ Esta disposición es derogada en el mes de Marzo de $1942^{32}$.

La justificación social y política que se dio a los ejercicios gimnásticos basada en el orden y la responsabilidad, no fue suficiente para lograr una cobertura hacia la mayoría de la población. No obstante, las prácticas se complementaban con discursos y expresión de propósitos sociales, muchas veces grandilocuentes, y alejados de la realidad cotidiana de las gentes, que crearon un imaginario colectivo de las demostraciones gimnásticas y los espectáculos deportivos.

\section{Ideal práctico. Nuevas habilidades y destrezas}

Las transformaciones económicas, relacionadas con los procesos de urbanización y nuevas formas de organización social, exigen habilidades y destrezas en distintos órdenes del modo de vivir. Por un lado respecto a las técnicas laborales que introducen la naciente industria, el comercio y la distribución. Por otro, las formas de uso del tiempo libre, el ocio y relación con los demás en espacios públicos y privados. La educación física se presenta como una estrategia para orientar la formación de estas realidades de orden práctico. El cuerpo sirve de medio y fin de las nuevas exigencias sociales. El ideal práctico no se realiza solamente con un fin utilitario inmediato, sino que se constituye en un campo de expresión lúdica y deportiva, sin pretender más satisfacción que la producida por su vivencia.

La educación física persigue el perfeccionamiento de todas las aptitudes en el orden físico, psíquico y moral, a fin de hacer rendir al hombre el máximo de energías con el mínimo de desgaste en beneficio personal o colectivo. Debe atender en forma metódica y

\footnotetext{
${ }^{30}$ NANNETI, Guillermo. (1939) Informe del Ministerio de Educación. Bogotá

${ }^{31}$ Decreto 1911 de 1940 y 1247 de 1941.

${ }^{32}$ Decreto 668 de 1942. 
progresiva a cada individuo; asegurar su desarrollo físico, armónico e integral y aumentar las resistencias orgánica; debe asegurar las aptitudes para la buena y correcta ejecución de todos los ejercicios naturales y de las prácticas utilitarias de aplicación; debe, en fin, desarrollar la energía y todas las cualidades adquiridas (físicas y viriles) hacia una idea dominante: el altruismo.

El desarrollo del ideal práctico produce diferentes transformaciones. Se generan nuevos movimientos corporales, cambia el paisaje urbano por la construcción y acomodación de escenarios deportivos y recreativos y cambia también el modo de vida de quienes practican actividades físicas como medio de satisfacción personal. El cuerpo se seculariza y se potencia como medio y fin cultural y social. Se abre paso a la homogenización desde modelos y modas provenientes de países considerados como centros de la cultura y el progreso.

En el contexto de la escuela activa la educación física alcanza su espacio de participación en la formación dc habilidades y destrezas necesarias a las nuevas realidades económicas y sociales, a través de la ejercitación sobre prácticas gimnásticas, deportivas, de juego y excursión. La aplicación de la educación física se hace sobre la necesidad de conocer el niño y las dos fuerzas que pugnan en su formación: la herencia y el medio natural y el medio humano ${ }^{33}$. Junto con la estructura mental se tiene en cuenta la física, pues se sabe de la influencia del medio y de la herencia sobre la fisiología de la persona para formar la individualidad. Con base en estas ideas se promueven el conocimiento psicofisiológico del niño y formas de enseñanza activa.

Se enseña jugando, paseando, refiriendo cuentos, poniendo a los alumnos en contacto con la naturaleza... La educación es integral. Los conocimientos van llegando por observación, por asociación, despertando el interés del niño por sistemas hechizantes, al propio tiempo que se van estudiando sus reacciones, analizando sus tendencias, observando su conducta. La educación moral, la educación intelectual, la educación física, marchan paralelamente ${ }^{34}$.

El énfasis fisiológico se mantiene como una necesidad para una política de educación por parte del Ministerio de Educación Nacional. "Si el Estado no puede ofrecer por el momento a esas masas auxilio intelectual y espiritual distinto al que se deriva de los cuatro años de la escuela primaria, está en el deber irrenunciable de hacer de ellos una etapa de preparación intensa que permita al futuro ciudadano defenderse con las mejores armas posibles de las contingencias adversas que le esperan en lo físico, en lo moral, en lo económico y en lo social”35

\begin{abstract}
En esa escuela debe proponerse en primer término la restauración fisiológica del niño y ya vimos por cuáles medios puede hoy procurarla. Y debe precaverlo, para la enseñanza de la higiene y el hábito de sus preceptos, de los riesgos que correrá en el futuro si abandona esas costumbres nuevas y olvida aquellas enseñanzas. La modificación del régimen alimenticio, la costumbre del baño, el uso del cepillo de dientes y del calzado, la familiarización del niño con su propio cuerpo, la extensión de la gimnasia y el deporte son hoy preocupaciones centrales de la escuela nueva y extremos de su programa que el Gobierno impulsa con su autoridad, con sus recursos económicos y con su desvelada simpatía ${ }^{36}$.
\end{abstract}

Cambia la idea de la educación física como actividad corporal a una concepción técnico científica que hacía énfasis en las prácticas y sus efectos en el individuo. La

\footnotetext{
${ }^{33}$ BEJARANO, Jorge. La Educación Física. Op.cit. pp. 35, 66-72

${ }^{34}$ Inspección Nacional de educación Primaria y Normalista. (1932). El doctor Decroly en Colombia, Imprenta

Nacional, Bogotá.

${ }^{35}$ ECHANDÍA, Darío. Op. Cit pp. 74-75

${ }^{36}$ ECHANDÍA, Darío. Ibid pp. 74-75 
metodología seguía un orden fisiológico y anatómico con un procedimiento a través de pasos estrictamente reglamentados, que implicaba una acción consciente y directa para el control de la persona ${ }^{37}$. En el transcurso del desarrollo de la educación física, el énfasis sobre las técnicas será más fuerte que el énfasis sobre el individuo. Se concibe al ser humano como objeto de adiestramiento específico hacia finalidades de carácter laboral y social, que coincide con un momento de diferenciación dc los campos de la ciencia en Colombia.

La visión activa, técnica y con fundamentos científicos promueve la utilización de diferentes medios de la educación física, en particular la gimnasia, el juego, el atletismo, el deporte y las excursiones para consolidar propósitos de identidad nacional, restauración fisiológica y adiestramiento técnico. La búsqueda de la economía de movimiento se basa en el perfeccionamiento del sistema nervioso, la coordinación y la educación de las sensaciones y los gestos. Estas prácticas desarrollan nuevos conceptos y comportamientos sociales. Requieren de nuevos escenarios para su realización así como de personas especializadas para su enseñanza, organización y dirección.

\section{Reflexiones finales}

El análisis de las relaciones entre educación física y cultura política en la primera mitad del siglo XX permite apreciar su articulación en la formación dcl individuo, del orden social del modelo económico, en el marco de las condiciones propias del proceso de modernización. De acuerdo con ello, se puede afirmar:

1. El ideal del ser humano integral, mens sana in corpore sano, se desarrolla con diferentes tipos de interés y de interpretación, pero concentra con mayor grado de amplitud las distintas formas de concebir la educación física. Desde la perspectiva espiritual para la cual el cuerpo es un mediador o para el punto de vista médico biológico, la necesidad de un cuerpo sano y una mente sana es un interés compartido.

2. En el ideal de mente sana en cuerpo sano, hay un énfasis formativo hacia el fortalecimiento moral a través del cuerpo, que se orienta a la restauración fisiológica que centra su atención en los principios orgánicos y fisiológicos, apoyados en la ciencia. Por ello este ideal integra el discurso de la salud que no se refiere solamente a la salud física y mental sino también a la salud moral y social; el discurso de la raza desde su lado optimista de fortalecimiento o desde su lado negativo de degeneramiento; el discurso moral e intelectual del progreso social, la fundamentación psicológica de las capacidades físicas y los fundamentos fisiológicos de la psicología.

3. El ideal cívico y normativo se realiza a través del uso de las formas de organización para la práctica de actividades físicas tanto en el espacio individual como en el espacio social. A partir de la disposición de los individuos en formaciones masivas y en marchas, se fue conformando un ceremonial de culto a los símbolos patrios y de reconocimiento y respeto a los representantes del orden social, autoridades civiles, eclesiásticas y militares integradas a las fiestas patrias, religiosas y cívicas.

4. En el contexto técnico práctico se producen cambios en la orientación de la educación física como un nuevo campo de la cultura formado sobre las bases de ideales políticos y educativos en los que se entrecruzan las preocupaciones por la salud, la

\footnotetext{
${ }^{37}$ Ibid pp. $74-75$ 
nación y el desarrollo productivo. El aprendizaje de disciplinas de movimiento aplicadas a diferentes situaciones sociales, laborales o estrictamente deportivas va dando respuesta a la formación de ideal práctico que orienta la preparación de nuevas habilidades y destrezas, en correspondencia con nuevas formas de vida y convivencia social.

5. A lo largo de cincuenta años se constituyó una nueva realidad cultural y social, que transformó los espacios escolares y sociales con la construcción de escenarios deportivos y recreativos y la modificación de la arquitectura escolar permitiendo a la vez que una mayor amplitud, la paulatina esquematización de los espacios en la mediada en que se asumían las especificaciones de los reglamentos deportivos. Como elemento nuevo en el espacio urbano hace su aparición social el estadio, como centro del espectáculo y de la sociedad de masas. La masificación del espectáculo deportivo produjo la necesidad de grandes inversiones en las ciudades sedes de los juegos, y con ello la articulación de intereses de diferente naturaleza alrededor del deporte y la cultura física.

6. La organización de la educación física muestra el desplazamiento progresivo entre ideales formativos de carácter amplio hacia propósitos técnicos y utilitarios. De una comprensión global como estrategia de formación social y nacional de las primeras décadas del siglo $X X$, se pasa progresivamente a una concepción de educación física mas centrada en las prácticas y técnicas, desde las cuales se influye en la totalidad. Ello hace posible que prácticas como el deporte, se transformen de medios en fines.

7. En la educación física se expresa el proceso de emancipación de la mujer, que de ser objeto de cuidado y esmero para cumplir su rol maternal y familiar, encuentra un espacio inicialmente restringido y regulado, para expresar sus potencialidades y acceder a prácticas antes vedadas para ella. Las distintas prácticas de la educación física, aún con las limitaciones, fueron un espacio para la secularización del cuerpo femenino, y la apertura a una mentalidad más abierta en la que se reconocen las capacidades de la mujer en el desempeño de distintas responsabilidades sociales, y la necesidad de una nueva actitud masculina. Pese a estos avances se mantuvieron las tensiones que enfrentaban los distintos puntos de vista sobre el desenvolvimiento de la mujer en la sociedad.

8. Estos cambios no universalizaron la cultura como ideal de la modernización pues se mantuvieron las diferencias, por ejemplo, entre la escuela rural y urbana, la enseñanza industrial y enseñanza académica, los colegios para las élites y los colegios populares, educación para los hombres y la educación para las mujeres; entre los alfabetizados y los analfabetas. Hacia 1918 el promedio de alfabetización estaba en el 32.5\% lo que significa cerca del $68 \%$ de analfabetismo, más acentuado en el sector rural que el urbano La población de más de 15 años con analfabetismo absoluto, en 1938 alcanzó el 44\% que corresponde a 2.223 .422 personas, de una población de $5.044 .100^{39}$. Los contenidos educativos son impartidos de manera desigual, manteniendo la diferenciación económica y social, y urbano rural, y con ello, poniendo límites y obstáculos a las posibilidades de acceso democrático. Si la modernidad universaliza y privilegia lo público, en Colombia, lo público es determinado por los intereses particulares de las élites.

\footnotetext{
${ }^{39}$ Aline Helg. La Educación en Colombia 1918- 1957, Cerec, Bogotá, 1986 p.36 


\section{Bibliografía}

AGOSTI, Luis. (1974). Gimnasia Educativa, Madrid: GTS Ex libris.

ALVAREZ, Alejandro; CASTRO, Jorge Orlando y NOGUERA, Carlos Ernesto. (1998). Ciudad, educación y escuela (1930-1950): los casos de Bogotá y Medellín, Informe final de investigación, Bogotá.

BERNARD, Michel (1980). El cuerpo, Buenos Aires, Paidos. $\mathrm{XXI}$.

BEERMAN, Marshall (1991). Todo lo sólido se desvanece en el aire, Bogotá, Ed. Siglo

CASTELLANOS MELO, Guillermo. (1992). "Historia social de la cultura política en el contexto de la identidad y de la crisis colombiana 1948-1990", en: Revista Universidad INNCA de Colombia. No. 2, abril 2.

CHINCHILLA G., Víctor Jairo. (1998). "Educación Física en e/ proceso de modernización" en: Memorias Encuentro de investigadores. Sao Paulo, Universidad de Campinas.

CUCUZZA, Héctor Rubén (1996). Historia de la educación en debate, Buenos Aires, Niño y Dávila Editores.

DOSSE Francois: La historia en migajas. Citado por VALENCIA, Alfonso el Magnánimo. (1988). "Anales" a la "Nuevaa Historia".

ELIAS, Norbert y DUNNING, Eric. (1995). Deporte y ocio en el proceso de la civilización, México, FCE.

FERREIRA, Neto (1997). AMARILIO et al, Pesquisa histórica na Educao Física, Vitoria, CEFD UFES.

FEIU MORENO, Paz. "Higiene, jardinería, tomate natural", en: Archipiélago $N^{0} 15$. 1995, pp 61-65.

FONTANA, Josep (1992). La historia después del fin de la historia, Barcelona, Crítica.

GALV1S RAMIREZ, Alberto. (1996). Oro plata y bronce: una aproximación a la historia del deporte colombiano. Bucaramanga.

GOMEZ MORENO, Alberto y PARRA PARRA, Luis Alberto (1986). Cincuenta años de la educación física colombiana como profesión 1936-1986. Bogotá, Publicaciones Universidad Central.

HELG, Aline (1987). La educación en Colombia 1918-1957: una historia social, económica y política. Bogotá, CEREC.

HERRERA, Martha Cecilia y LOW Carlos (1994). Los intelectuales y el despertar cultural del siglo. El caso de la Escuela Normal Superior: una historia reciente y olvidada. Bogotá, Universidad Pedagógica Nacional. 
HERRERA, Martha Cecilia. Historia de la Educación en Colombia: La República Liberal y la modernización de la Educación: 1930-1946, en: Revista Colombiana de educación.

HERRERA, Martha Cecilia (1997). Educación y cultura política en América Latina: una aproximación histórico social. Bogotá. Maestría Historia de la Educación. UPN.

HERRERA, Martha Cecilia. (1999). Modernización y Escuela Nueva en Colombia. Bogotá. Universidad Pedagógica. Plaza y Janés.

HOBSBAWM, Eric. Sobre la historia (1998). Barcelona, Grijalbo Mondadori.

LANGLADE, Alberto y REY, Nelly (1970). Teoría general de la gimnasia, Buenos Aires, Stadium.

LLCHNER, Norbert et al. (1987) Cultura política y democratización, Buenos Aires, CLACSO.

ed. México, FCE.

(1995). Los patios interiores de la democracia: subjetividad y política, 2a

LOPEZ DE LA ROCHE, Fabio (1997). Aproximación al concepto de cultura política. Bogotá.

MARTINEZ, Luis Ignacio (1997). Surgimiento del deporte y el tiempo libre en Bogotá 1895-1920, 220 p. Trabajo de grado (licenciado en educación física). Universidad Pedagógica Nacional. Facultad de Ciencia y Tecnología. Departamento de educación física.

MAUSS, Marcel. Técnicas y movimientos corporales. En: Sociología y Antropología. p 337-356

MELO, Jorge Orlando. (1994). Algunas consideraciones globales sobre "Modernidad "y "Modernización" en Colombia: el despertar de la modernidad, Bogotá, Ediciones Foro Nacional por Colombia.

MINISTERIO DE EDUCACION NACIONAL. (1995). Elementos para el análisis del desarrollo de la educación física en Colombia. Bogotá, Serie Guías MEN.

MOLANO B, Alfredo y VERA G, César (1982). Evolución de la política educativa en el siglo XX. Primera parte: 1900-1 958. Bogotá. Universidad Pedagógica.

QUESADA, Patricia y ALONSO SÁNCHEZ, Néstor. (1990). La educación físiica en Colombia: una historia por construir Cali. 119 p. Trabajo de grado (Licenciado en educación física) Universidad del Valle. Facultad de Educación. Departamento de educación física y deportes.

QUICENO, Humberto (1998). Pedagogía católica y escuda activa en Colombia 19001935. Bogotá, Foro Nacional por Colombia.

RODRIGLJEZ, Bibiana Astrid (1997). La educación física y la mujer: 1936-1950: Aproximación a una historia de la sexualidad. Bogotá, 170 p. Trabajo de grado (licenciada en educación física) Universidad Pedagógica Nacional. Facultad de educación física. 
SÁENZ OBREGON, Javier; SALDARRIAGA, Oscar y OSPINA, Armando (1997). Mirar la infancia: pedagogía, moral y modernidad en Colombia. Medellín, Colciencias. Foro Nacional por Colombia. Universidad de Antioquia. 2 vol.

SOARES, Carmen. (1994). Educao Física: Raízes européias e Brasil. Campinas. Edit. Autores Asociados.

Autores Asociados.

(1998) Imagens da Educao no corpo. Campinas. Edit.

THOMPSON, E. P. (1995). Costumbres en común: Crítica. Barcelona. (Grijalbo Mondadori).

TORRES, Iván; PEÑA, Clara y Chinchilla, Víctor. et al. (1 996). De qué ha de ocuparse la educación física? Horizonte de sentido: De cómo llegamos a ser lo que somos. Bogotá. 135 p. Trabajo de grado. (Especialistas en pedagogía y didáctica dc la educación física). Universidad Pedagógica Nacional. Facultad de educación física.

TOVAR ZAMBRANO, Hermes et al. (1997). Pensar el pasado. Bogotá. Archivo General de la Nación y Universidad Nacional, 192 p.

TURNER, Bryan S. (1994). El cuerpo y la sociedad. Exploraciones en teoría social México, FCE

VACA HERNÁNDEZ, Ángel Humberto (1987). Historia de la educación física colombiana a través de sus normas. Bogotá. Universidad Central.

(1996). Historia del alma mater de la educación física en Colombia. Bogotá, Universidad Pedagógica Nacional.

ZEIGLERF, Earle (1979). History of physica/ Education and Sport. Englewood Ciiff, N J, John E. Nixon Series Editor.

\section{ARCHIVOS}

Archivo General de la Nación.

Biblioteca Luis Ángel Arango.

Asociación Colombiana de Profesores de Educación Física.

Comité Olímpico Colombiano.

Maestría Historia dc la Educación y la Pedagogía, Universidad Pedagógica. 